\title{
iTRAQ-based Comparative Proteomic Analysis of Flag Leaves of Two Wheat (Triticum aestivum L.) Genotypes Differing in Waterlogging Tolerance at Anthesis
}

\author{
Mingmei Wei \\ Yangtzeu University \\ LiuLong Li \\ Yangtze University \\ Ke Xie
}

Beijin University of Science and Technology

Rui Yang

Yangtze University

Xiaoyan Wang

Yangtze University

Aihua Sha ( $\square$ aihuasha@yangtzeu.edu.cn )

Yangtze University

Research article

Keywords: Wheat; Waterlogging; Proteomic; SPAD; qRT-PCR

Posted Date: December 23rd, 2019

DOI: https://doi.org/10.21203/rs.2.9778/v3

License: (c) (i) This work is licensed under a Creative Commons Attribution 4.0 International License. Read Full License 


\section{Abstract}

Background : Waterlogging is one of the major abiotic stresses limiting wheat product. Plants can adapt to waterlogging with changes in morphology, anatomy, and metabolism. Many genes and proteins play critical roles in adaptation to waterlogging. Results : the iTRAQ-based proteomic strategy was applied to identify the waterlogging-responsive proteins in wheat. A total of 7,710 proteins were identified in two wheat varieties, XM55 (waterlogging-tolerant) and YM158 (waterlogging-sensitive), at anthesis under waterlogging or not. Sixteen proteins were differentially accumulated between XM55 and YM158 under waterlogging with cultivar specificity. Of these, 11 proteins were up-regulated and 5 proteins were down-regulated. The up-regulated proteins included Fe-S cluster assembly factor, heat shock cognate 70, GTPbinding protein SAR1A-like, and CBS domain-containing protein. The down-regulated proteins contained photosystem II reaction center protein $\mathrm{H}$, carotenoid 9,10 (9',10')-cleavage dioxygenase-like, psbP-like protein 1 , and mitochondrial ATPase inhibitor. In addition, 9 proteins were responsive to waterlogging with non-cultivar specificity. These proteins included 3-isopropylmalate dehydratase large subunit, solanesyl-diphosphate synthase 2, DEAD-box ATP-dependent RNA helicase 3 , and 3 predicted or uncharacterized proteins. Conclusion s: This study revealed that the proteins were differential accumulated between the two contrast waterlogging wheat varieties in response to waterlogging, which provide valuable insights into wheat response to waterlogging stress. These differentially accumulated proteins might be applied to develop waterlogging tolerant wheat in further breeding programs.

\section{Background}

Waterlogging $(\mathrm{WL})$ is a major abiotic stress caused by high rainfall, irrigation practices and/or poor soil drainage [1, 2]. Anoxic soils and severe hypoxia or anoxia within roots often result from WL [2], which affects several physiological processes such as water absorption, hormone relation, ion uptake and transport, and superoxide dismutase activities [3].

Plants can adapt to WL with changes in morphology, anatomy, and metabolism [4]. Development of a shallow root system and formation of aerenchymatous adventitious roots are the main morphological/anatomical changes [5, 6], and are controlled by plant hormones such as ethylene, auxin, abscisic acid (ABA), cytokinin, jasmonates (JAs), and gibberellin (GA) [4]. In rice, lysigenous aerenchyma and a barrier to radial $\mathrm{O}_{2}$ loss form in roots to mitigate WL stress by supplying $\mathrm{O}_{2}$ to the root tip [7].

In the past years, great efforts have been made to investigate the mechanism of WL tolerance at the molecular level. Many genes have been demonstrated to mediate WL in cotton [8, 9], rapes [10], maize [11, 12], cucumber [13]. Previous studies showed that 52 and 146 proteins were differentially expressed in tomato leaves and cucumber adventitious roots in response to WL stress, respectively $[14,15]$. It has been demonstrated that 100 proteins were responsive to WL stress in different tissues of WL-sensitive and WL-tolerant barleys [16]. Over-expression of the Kiwifruit AdPDC1 (Actinidia deliciosa pyruvate decarboxylase 1) could enhance WL resistance in transgenic Arabidopsis thaliana [17].

Wheat (Triticum aestivum L.) is one of the most economically important cereal crops in the world. WL has reduced wheat grain yields by about $20-50 \%$ in the UK, North America, and Australia [18]. Some attempts have been made to investigate the regulation mechanism responding to $W L$ in wheat. Transcripts of phenylalanine ammonia-lyase 6 , cinnamoyl-CoA reductase 2, ferulate 5-hydroxylase 2 are involved in lignin biosynthesis, and have been shown to be repressed by WL [19]. Genes regulating metabolism of hormones change under WL, which include ACS7 and ACO2 for ethylene biosynthesis, TDC, YUC1, and PIN9 for indole acetic acid (IAA) biosynthesis/transport, LOX8, AOS1, AOC1, and JAR1 for JA metabolism, GA3ox2 and GA2ox8 for GA metabolism, IPT5-2, LOG1, CKX5, and ZOG2 for cytokinin metabolism, NCED1 and NCED2 for ABA biosynthesis [4]. Anoxia under WL reduces the abundance of denitrification gene nirS in the rhizosphere of wheat [20]. Besides, some proteins, including acid phosphatase, oxidant protective enzyme, SAM1, play 
critical roles in the adaptation of WL and hypoxia stress in wheat [21]. However, understanding of the molecular basis of WL tolerance is still limited in wheat.

WL has become a major constraint for wheat production in southeast of China due to excessive rainfall during the growing season, which is especially severe during the critical grain formation periods of anthesis and maturation [18,22]. Proteomics is a useful and important method for investigating crop responses to stress by detecting changes in expression and post-translational modification of proteins [23]. Proteomic techniques have been performed to investigate proteins in response to WL in tomato [14], soybean [24], cucumber [15], barley [16], etc. Proteomic approaches have also been successfully used to perform proteomic profiles in response to flooding, drought, high temperature, salt, metal stresses in wheat [25]. In the present study, we utilized proteomics to identify proteins in response to WL in two wheat varieties with different $\mathrm{WL}$ tolerances aiming at clarifying the underlying regulation mechanisms of the WL response in wheat. In the further studies, this study will serve as a resource for the development of WL tolerant wheat varieties.

\section{Results}

\section{Phenotypic and Physiological analysis of two varieties}

WL is known to induce chlorosis and early senescence of leaves [26]; Firstly, we detected the chlorophyll concentration in expanded flag leaves of WL-tolerant variety XM55 and WL-sensitive variety YM158 by measuring SPAD (soil-plant analysis development) at the anthesis stage. The SPAD value of XM55 was higher than that of YM158 during 0-7 days, and it was less than or equal to that of YM158 during 7-21 d under WL (Fig. 1A). However, the SPAD value of XM55 was higher than that of YM158 between 0-21 days under normal conditions (CK). The SPAD value of XM55 under normal conditions decreased below that of WL treated XM55 after $7 \mathrm{~d}$, whereas it was decreased below that of YM158 under CK at $5 \mathrm{~d}$ (Fig. 1A). The reductions of SPAD in XM55 from 0 to $7 \mathrm{~d}, 7$ to $14 \mathrm{~d}$, and 14 to $21 \mathrm{~d}$ under WL were $2.7 \%, 4.2 \%$, and $7.8 \%$, whereas they were $4.7 \%, 6.9 \%$, and $13.4 \%$ in YM158, respectively.

Soil WL causes serious hypoxia in plant roots, obstructs root growth and development, decreases root activity, and decreases root water permeability; this affects plant water uptake and transpiration rate, thereby leading to water deficit in plants and alterations in the above-ground distribution of water [26, 27]. We also measured the above-ground water contents in the two varieties. Under WL, the water contents in flag leaves, ears, and stem and sheath were significantly higher in XM55 than in YM158 from 7 to $21 \mathrm{~d}$, whereas this pattern occurred from 14 to $21 \mathrm{~d}$ under CK (Fig. 1B, 1C, 1D)

WL at elongation or post-anthesis is known to affect grain yield, as well as accumulation and remobilization of dry matter in wheat [27]. We measured the changes of aboveground dry matter accumulation (DMA), yield, and yield-related traits of the two varieties. WL had different effects on XM55 and YM158. The DMA at anthesis (DMA1) before WL were roughly similar between XM55 and YM158, but under WL, DMA values were decreased by $12.5 \%$ and $20.5 \%$ in XM55 and YM158 relative to the CK control at the mature stage, respectively (Table 1). At the same time, kernels per spike, 1000kernel weight, grain yield weight, and harvest index were decreased under WL by 3.3, 18.1, 26.2 and 15.9\% in XM55, and by 10.8, 36.2, 36.8 and 21.8\% in YM158 relative to their CK control values, respectively (Table 1). Clearly, WL had greater effects on YM158 instead of XM55, especially the 1000-kernel weight and grain yield weight. Overall, it could be inferred that XM55 showed better WL tolerant than YM158.

\section{Waterlogging Induced Proteome Change in XM55 and YM158}

To further explore the molecular mechanisms that mediate different responses to WL, iTRAQ method was used to analyze proteome changes in flag leaf of both cultivars. After protein extraction, enzyme digestion, iTRAQ labeling, equal mixing and SCX pre-separation, all samples were subjected to LC-MS/MS in three independent replicates. In the present study, a total of $1,087,846$ spectra were detected, among which, 37,952 could be matched and 55,206 were unique 
spectra, and 37,985 peptides could be identified with 19,279 being unique peptides, and 7710 proteins were identified (Fig. 2A); the proteins identified in the flag leaf of the XM55 and YM158 plants were supported by unique peptides. Of those proteins, $54.0 \%(4,164)$ were inferred from more than three unique peptides (Fig. 2B).

\section{Pairwise comparison of protein profiling in XM55 and YM158 under WL}

To identify differentially expressed proteins (DEPs) between the two cultivars in response to WL, proteins with more than a 1.2-fold change in abundance $(p<0.05)$ between XM55 and YM158 under WL and CK were investigated. Based on this criterion, 23 DEPs (14 up-regulated and 9 down-regulated) showed differential expressed between XM55 and YM158 under WL (XM55-WL/YM158-WL), and 52 DEPs (31 up-regulated and 21 down-regulated) were differently expressed between XM55 and YM158 under CK (XM55-CK/YM158-CK) (Fig. 3). At the same time, 7 proteins (i.e., TRIAE_CS42_2BL_TGACv1_130584_AA0414140.1, TRIAE_CS42_2BL_TGACv1_131439_AA0427700.2, TRIAE_CS42_4BL_TGACv1_321826_AA1065960.1, TRIAE_CS42_2BL_TGACv1_132610_AA0438610.1, TRIAE_CS42_6BL_TGACv1_503168_AA1627380.1, TRIAE_CS42_6BL_TGACv1_503168_AA1627380.2, TRIAE_CS42_6BL_TGACv1_503168_AA1627380.3) were differentially accumulated in both (XM55-WL/YM158-WL) and (XM55-CK/YM158-CK), which might indicate cultivar specific protein accumulation irrespective of WL treatment (Fig. 3, Table 2, Table S1). Excluding these 7 overlapping DEPs, a total of 16 DEPs were remained between XM55 and YM158 under WL (Fig. 3B); of these, 11 DEPs were up-regulated, including members of Fe-S cluster assembly factor, heat shock cognate $70 \mathrm{kDa}$ protein, GTP-binding protein SAR1A-like, and CBS domain-containing protein, respectively. The 5 downregulated proteins were photosystem II reaction center protein $\mathrm{H}$, carotenoid 9,10 $\left(9^{\prime}, 10^{\prime}\right)$-cleavage dioxygenase-like, psbPlike protein 1, and mitochondrial ATPase inhibitor (Table 2).

\section{Proteomic dynamics in XM55 and YM158 between WL and CK}

The DEPs in XM55 and YM158 between WL and CK were identified. There were 84 DEPs (35 up-regulated and 49 downregulated) between XM55-WL and XM55-CK, and 59 DEPs (13 up-regulated and 46 down-regulated) between YM158-WL and YM158-CK (Fig. 3, Table S2, Table S3). Most proteins responsive to WL were specific to XM55 or YM158. However, 9 proteins were differentially expressed in both XM55-WL/XM-CK and YM158-WL/YM158-CK (Fig. 3, Table S2, Table S3), which might be WL responsible proteins with non-cultivar specificity. These proteins were 3-isopropylmalate dehydratase large subunit, solanesyl-diphosphate synthase 2, DEAD-box ATP-dependent RNA helicase 3, and three predicted or Uncharacterized proteins. 3-isopropylmalate dehydratase catalyses, the stereo-specific isomerization of 2isopropylmalate and 3-isopropylmalate participate in the biosynthesis of leucine. Meanwhile, we compared these DEPs with those identified from the previous study by Pan et al. which investigated proteins related to waterlogging stress. Importantly, several key DEPs, including S-adenosylmethionine synthetase 2, DEAD-box ATP-dependent RNA helicase 3, peroxidase, thaumatin-like protein, were observed in both two studies.

\section{Functional Categorization, GO and KEGG pathway Enrichment Analysis of the DEPs}

The functional information of all differentially accumulated proteins in Fig. 3 were obtained by searching against the UniProt-GOA database, which were assigned to three categories based on GO annotation, that is, cellular compartment, biological process, and molecular function. The differentially expressed proteins among XM55 and YM158 under WL belonged to eight biological processes, 11 cellular compartments, and two different molecular functions (Fig. 4, Table S4). In terms of biological processes, metabolic process, cellular process and cellular component organization or biogenesis were the three major groups. It was suggested that the DEPs may be involved in primary metabolic processes, and these impart differential WL tolerances to XM55 and YM158. Cell, cell part, and membrane-enclosed lumen were the top three cellular compartments, implying that various changes in cell structure had effects on tolerance to WL among different varieties. Binding was the major molecular functional groups, and a small amount of differentially accumulated proteins were involved in catalytic activity, which showed that protein binding affects tolerance to WL. 
The differentially expressed proteins among XM55 or YM158 under WL and CK belonged to 11 or 8 biological processes, 9 or 11 cellular compartments, and 6 or 3 molecular functions (Fig. S1, Fig. S2, Table S4), respectively. Metabolic process, cellular process, and single-organism process were both the three major biological processes. Cell, cell part, and organelle were both the top three cellular compartments. Catalytic activity and binding were both the two-major molecular functional groups. Those results indicated that primary metabolic processes, cell structure, and catalytic activity were generally affected by WL regardless of cultivar tolerance.

To characterize the functional consequences of the differentially expressed proteins associated with WL, the enriched pathways were assigned based on KEGG terms. The results indicated that the proteins related to terpenoid backbone biosynthesis, amino sugar and nucleotide sugar metabolism, and fructose and mannose metabolism were affected by WL in XM55, whereas terpenoid backbone biosynthesis and fatty acid biosynthesis were affected in YM158.

Tuberculosis and RNA degradation were affected by WL both in XM55 and YM158 (Table S5).

\section{Correlation of DEPs with mRNA Expression}

To verify the correlation between the expression levels of the differentially expressed proteins and their mRNAs, the mRNA expression levels of 28 differentially expressed proteins were analyzed using qRT-PCR method (Table S6). Among them, 16 genes exhibited consistent expression patterns with their proteins, whereas 12 showed discrepancies between protein accumulation and mRNA expression (Fig. 5). The discrepancy between protein accumulation and mRNA expressions might be ascribed to translational and posttranslational regulatory processes or feedback loops between the processes of mRNA translation and protein degradation [28]. These results were consistent with previous studies that transcription patterns do not always directly correlate with protein expression levels $[16,29,30]$. Meanwhile, the expression

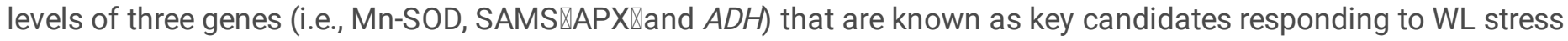
were investigated. The results showed that SAMS and APX were gradually up-regulated in YM158 compared with control group after WL treatment, and their expression reached the highest levels at 5 days in YM158 after WL treatment (Fig. S3). The peak of expression levels of Mn-SOD was observed at 5 days after treatment in both two varieties. ADH1A and ADH1D were not detected to express in both varieties under our experiment (data not shown).

\section{Discussion}

Wheat is negatively affected by waterlogging stress [31,32], and anthesis is the most sensitive stage [33], during which, waterlogging usually occurs. In this study, we compared the waterlogging tolerance of two wheat varieties, and found that waterlogging impacted the chlorophyll content, water content, grain weight and its components, and accumulation of dry matter after anthesis in both varieties at anthesis. Notably, the degree of waterlogging influence varied among different wheat varieties, which could explain why XM55 was less sensitive to water stress than YM158 in this study.

In the past years, many proteomic studies involved in WL stress of plants have been reported, such as Alfalfa (Medicago sativa) [34], barley (Hordeum vulgare L.) [35], tomato [36] and soybean [37], and these studies have reported some putative proteins that may play key roles in response to WL stress. For instance, the proteins of oxygen-evolving enhancer protein 1, ATP synthase subunit and heat shock protein 70 were up-regulated in WL tolerant genotype of Alfalfa, which are related to photosynthesis, metabolism and energy. In this study, iTRAQ and HPLC-MS techniques were used to analyze protein expression patterns of flag leaf which could reveal the effect of waterlogging stress on during anthesis in two different varieties (i.e., XM55 and YM158). We found that the number of DEPs identified in the pairwise comparison was relatively lower than the previous studies [38,39], inferring that the different tissues (e.g., leaf, root, stem) and number of biological replicates could determine the number of DEPs to some extent. Meanwhile, the treatment and sampling stage in the experiment design could also decrease the difference of genetic and proteomic expression. For example, Pan et al. [21] investigated the proteomic patterns of seedling roots under hypoxia conditions in two wheat 
genotypes (S and T), and the results showed that hundreds of DEPs were detected in the comparisons except that (33 DEPs) in T (CIGM90.863) at 1 day. On the other hand, these candidate proteins and pathways identified from pairwise comparisons in this study were effective subjects for us to investigate proteomic regulation of WL tolerance in wheat. A total of 11 up-regulated proteins in XM55 were identified in response to WL that were involved in iron acquisition, proteins folding assistant, cargo secretion, abiotic stresses, whereas 5 proteins were down-regulated, which participated in light energy usage, strigolactone biosynthesis, vesicle-mediated secretion. The different tolerance of waterlogging between XM55 and YM158 might be ascribed to those differentially accumulated proteins. In detail, the DEPs related to Fe/S clusters participate in diverse cellular processes in almost all organisms, which include respiration, metabolism, DNA replication and repair, and regulation of gene expression $[40,41]$. The gene sufT, which is involved in the Fe/S cluster assembly pathway, has been reported that it is necessary for effective symbiosis to enhance iron availability [42]. Heat shock cognate $70 \mathrm{kDa}$ protein is a chaperone which assist the folding of other proteins in vivo, and it was found to have increased expression in sugarcane plant subjected to WL [43]. Sar1 in plants plays roles in GTPase activity, cargo secretion, membrane constriction, etc. [44]. Over expression of CBS domain-containing protein could enhance tolerance to different abiotic stresses in tobacco [45] and soybean [46]. These proteins were up-regulated in XM55 compared to YM158 under WL, indicating that their enhanced accumulation may be responsible for WL tolerance. Interestingly, some potential key proteins which have been considered as critical factors for WL tolerance in wheat were not differentially expressed in this study. For instance, Wang et al. [38] revealed that S-adenosylmethionine synthtase (SAMS), involved in ethylene biosynthesis pathway, was upregulated by WL stress in wheat. Likewise, the alcohol dehydrogenases participating in carbohydrate metabolism exhibited upregulation with WL stress in plant $[21,37,47,48]$. Notably, these key candidates were not differentially expressed in flag leaf between control and WL treatment groups, inferring that these genes exhibited tissue-specific, and probably they were highly expressed in root or other tissues instead of flag leaf of wheat.

Photosystem II (PSII) reaction center protein $\mathrm{H}$ and psbP are constituents of PS II, which uses light energy to split water into chemical products [49]. Carotenoid cleavage dioxygenases (CCDs) cleave carotenes and xanthophylls to apocarotenoids, which may mediate strigolactone biosynthesis and are responsive to phosphorus deficiency [50], wounding, heat, and osmotic stress [51]. The ATPases play roles in diverse cellular activities such as vesicle-mediated secretion, membrane fusion, cellular organelle biogenesis, and hypersensitive responses (HR) in plants [52]. These proteins were down-regulated in XM55 compared to YM158 under WL, suggesting that WL tolerance might be associated with reduced energy production, changes of hormone content and cellular activities in plants.

In addition, 9 DEPs were detected in both WL tolerant and non-tolerant varieties (Fig. 3, TableS2, TableS3), which were involved in leucine biosynthesis, plastoquinone biosynthesis, and ribosomal structure remodeling, indicating they played basic roles in tolerance of WL stress. GO and KEGG pathway analysis indicated that proteins involving in primary metabolic processes, cell structure, protein binding determined the different tolerance to WL between XM55 and YM158. Compared with the control group, the proteins upregulated in WL group also play important roles in tolerance of WL stress. For instance, Solanesyl-diphosphate synthase 2 is involved in plastoquinone biosynthesis, which regulates gene expression and enzyme activities as a photosynthetic electron carrier, and plays a central photoprotective role as an antioxidant [53]. DEAD-box ATP-dependent RNA helicase 3 is involved in ribosomal structure and it was shown to be markedly suppressed after salt treatment in cotton [54]. These proteins were responsive to waterlogging without cultivar specificity, indicating that the leucine, reactive oxygen species, and the ribosome may play roles in basic defense to WL.

qRT-PCR analysis indicated that consistent expression patterns were observed between mRNAs and proteins for most selected proteins. However, a discrepancy was also identified for several proteins between protein accumulation and mRNA expression. It could be suggested that transcription patterns do not always directly correlate with protein expression levels $[16,29,30]$, which might be ascribed to translational and posttranslational regulatory processes or feedback loops between the processes of mRNA translation and protein degradation [28].

Page $6 / 20$ 


\section{Conclusions}

This study showed that many proteins were differential expressed between two waterlogging wheat varieties in response to WL stress. Some key proteins, such as Fe-S cluster assembly factor, heat shock cognate 70, GTP-binding protein SAR1A-like, and CBS domain-containing protein, may play important roles in responses to WL stress for wheat. Meanwhile, we found that WL stress could result in down-regulation of chlorophyll and the content of enzymes related to photorespiration in wheat, which can impact the synthesis of metabolic enzymes. This study provided novel insights into proteomic response to WL stress, and the DEPs might be applied to biological markers for developing waterlogging tolerant varieties of wheat in a breeding program.

\section{Methods}

\section{Plant growth conditions and treatments}

The wheat cultivars XM55 and YM158 were used in the screen for WL-responsive proteins. They were sown in the farm of Yangtze University located in Jingzhou, Hubei Province, China in growing season on November 15, 2017. The topsoil $(0-20 \mathrm{~cm})$ of the experimental field is a clay loam and the nutrient status was as follows, organic matter content was $10.5 \mathrm{~g} \cdot \mathrm{kg}^{-1}$; available $\mathrm{N}$ concentration was $33.41 \mathrm{mg} \cdot \mathrm{kg}^{-1}$; available $\mathrm{P}_{2} \mathrm{O}_{5}$ concentration was $45.37 \mathrm{mg} \cdot \mathrm{kg}^{-1}$; and available $\mathrm{K}_{2} \mathrm{O}$ concentration was $80.26 \mathrm{mg} \cdot \mathrm{kg}^{-1}$.

The field experiments were conducted with 2 groups; The WL treatment group consisted of wheat at anthesis treated with WL for 7 days, and wheat at anthesis without WL treatment served as the control group. Three replicates were performed per treatment for each variety, and the plot areas were $12 \mathrm{~m}^{2}(2 \mathrm{~m} \times 6 \mathrm{~m})$. At the sowing stage, the base application rate was $90 \mathrm{~kg} /$ hectare of pure nitrogen from the application of compound fertilizer, and the ratio of available nitrogen $\mathrm{N}$, phosphorus $\mathrm{P}_{2} \mathrm{O}_{5}$ and potassium $\mathrm{K}_{2} \mathrm{O}$ in compound fertilizer was 26:10:15. At the jointing stage, pure nitrogen was applied at $90 \mathrm{~kg} /$ hectare in the form of urea. At the trefoil stage, 224 plants $\mathrm{m}^{-2}$ remained. Otherwise, regular field management practices were employed. Wheat plants for both cultivars at anthesis stage were subjected to control and waterlogging treatment for $7 \mathrm{~d}$. When $50 \%$ plants begin to bloom, in which the plants height are more than $80 \mathrm{~cm}$, the plots were submerged in $2 \mathrm{~cm}$ - depth water as waterlogging treatment. A total of 10 plants was selected per group, and three biological replicates were conducted for each treatment. The flag leaves were collected, immediately frozen, and stored in liquid nitrogen for protein and RNA extraction for qRT-PCR.

\section{Protein extraction, digestion and iTRAQ labelling}

Total protein was extracted using the cold acetone method. Samples were ground in liquid nitrogen and dissolved in 2 $\mathrm{mL}$ lysis buffer (8 M urea, 2\% SDS, 1x Protease Inhibitor Cocktail (Roche Ltd. Basel, Switzerland). Subsequently, sonication on ice for $30 \mathrm{~min}$ and centrifugation at $13,000 \mathrm{rpm}$ for $30 \mathrm{~min}$ at $4^{\circ} \mathrm{C}$ were conducted. Proteins were precipitated with ice-cold acetone at $-20^{\circ} \mathrm{C}$, and the precipitate was cleaned with acetone three times and re-dissolved. The protein quality was determined by SDS-PAGE (Fig. S4).

Bicinchoninic acid assay (BCA; Pierce, MA, USA) was used to determine the protein concentration. The 100- $\mu \mathrm{g}$ protein from the previous step was transferred into a new tube and adjusted to a final concentration of $1 \mu \mathrm{g} / \mu \mathrm{L}$, and then treated with $11 \mu \mathrm{L}$ of $1 \mathrm{M} \mathrm{DTT}$ (DL-Dithiothreitol) at $37^{\circ} \mathrm{C}$ for 1 hour. Then we used $120 \mu \mathrm{L}$ of the $55 \mathrm{mM}$ iodoacetamide and incubated the mixture for 20 minutes at room temperature in the dark.

For each sample, proteins were precipitated with ice-cold acetone, then re-dissolved in $100 \mu \mathrm{L}$ TEAB $(0.25 \mathrm{M}, \mathrm{pH} 8.5)$. Then samples were tryptic digested with trypsin (Promega, Madison, WI) at $37^{\circ} \mathrm{C}$ for 4 hours (trypsin: protein $1: 100$ ). The 
resultant peptide mixture was labeled with iTRAQ tags 113 through 118. The labeled samples were combined and dried in vacuum.

\section{Strong cation exchange (SCX) fractionation and LC-MS/MS analysis}

The combined labeled samples were bound to a SCX fractionation column connected with a high performance liquid chromatography (HPLC) system. The peptide mixture was re-dissolved in the buffer A (20 mM ammonium formate in water, $\mathrm{pH} 10.0$ ), and then fractionated by high $\mathrm{pH}$ separation using Ultimate 3000 system (Thermo Fisher scientific, MA, USA) connected to a reverse phase column (Gemini-NX 3u C18 110A column, $2.0 \mathrm{~mm}$ x $150 \mathrm{~mm}, 3 \mu \mathrm{m}$, (Waters Corporation, MA, USA). High pH separation was performed using a linear gradient starting from $5 \%$ to $45 \%$ buffer $B$ (20 $\mathrm{mM}$ ammonium formate in $80 \% \mathrm{ACN}, \mathrm{pH} 10.0$ ) in $40 \mathrm{~min}$. The column flow rate was maintained at $0.2 \mathrm{~mL} / \mathrm{min}$ and column temperature was maintained at $30^{\circ} \mathrm{C}$. A total of 12 fractions were collected, and each fraction was dried in a vacuum concentrator for the next step.

Peptide fractions were resuspended with $30 \mu \mathrm{L}$ solvent $\mathrm{C}$ ( water with $0.1 \%$ formic acid), respectively, and separated by nanoLC and analyzed by electrospray tandem mass spectrometry. The experiments were performed on an Easy-nLC 1000 system (Thermo Fisher Scientific, MA, USA). A total of $10 \mu \mathrm{L}$ peptide sample was loaded onto the trap column (Thermo Scientific Acclaim PepMap C18, $100 \mu \mathrm{m} \times 2 \mathrm{~cm}$ ), with a flow of $10 \mu \mathrm{L} / \mathrm{min}$ for 3 min and subsequently separated on the analytical column (Acclaim PepMap C18, $75 \mu \mathrm{m} \times 15 \mathrm{~cm}$ ) with a linear gradient, from $3 \%$ to $32 \%$ solvent $D$ (ACN with $0.1 \%$ formic acid) in $120 \mathrm{~min}$. The column flow rate was maintained at $300 \mathrm{~nL} / \mathrm{min}$.

The fusion mass spectrometer was run in the data-dependent mode to switch automatically between MS and MS/MS acquisition. Survey full-scan MS spectra (m/z 350-1550) were acquired with a mass resolution of $120 \mathrm{~K}$, followed by sequential high energy collisional dissociation MS/MS scans with a resolution of $30 \mathrm{~K}$. The isolation window was set as 1.6 Da. MS/MS fixed first mass was set at 110. In all cases, one microscan was recorded using dynamic exclusion of 45 seconds.

\section{Database search and Quantification}

The mass spectrometry data were transformed into MGF (Mascot generic format) files with Proteome Discovery 1.2 (Thermo, PA, USA) and analyzed using Mascot software version 2.3.2 (Matrix Science, London, UK). Mascot database was set up for protein identification using Triticum aestivum $L$ database in NCBI nr (release 2017_03);

SwissProt/UniprotKB (release 2018_06) and International Protein Index (IPI; version 3.16). Trypsin/P was chosen as the enzyme with two missed cleavages allowed; Peptide tolerance was set at $10 \mathrm{ppm}$, and Mascot was searched with a fragment ion mass tolerance of $0.050 \mathrm{Da}$; a parent ion tolerance of 10.0 PPM. Significance threshold $p<0.05$ (with $95 \%$ confidence). The average values of the biological replicates were used to indicate the final protein abundances for each sample. The full list of proteins identified in this study is shown in supplementary Table S7. Proteins with a 1.2-fold change between samples and a $p$ value less than 0.05 were determined as differentially expressed proteins (DEPs).

\section{GO and KEGG Enrichment analysis}

The DEPs were selected for functional enrichment analysis. The hypergeometric test was used to determine significant enrichment of $\mathrm{GO}$ terms relative to the background. The $p$-value was adjusted with FDR Correction, setting FDR $\leq 0.05$ as a threshold. The GO terms with FDR $\leq 0.05$ were defined as significantly enriched GO terms. Likewise, KEGG pathway enrichment was also performed with KEGG database [55]. The calculated $p$ value was adjusted with FDR Correction, setting FDR $\leq 0.05$ as a threshold.

\section{RNA extraction and qRT-PCR}


Total RNA was extracted using the TRIZOL reagent (Invitrogen, Carlsbad, CA, USA). Then RNA samples were reversetranscribed using the RevertAid ${ }^{\mathrm{TM}}$ First Strand cDNA Synthesis Kit (Thermo Fisher Scientific, MA, USA) according to the manufacturer's protocol. Each reaction was conducted in $10 \mu \mathrm{L}$ mixture containing $5 \mu \mathrm{L}$ of SYBR green (SYBR@ Premix Ex Taq $^{\text {TM }}$ (TliRNaseH Plus), TAKARA, Japan), $0.6 \mu \mathrm{L}$ forward and reverse primers (10 $\left.\mu \mathrm{M}\right), 2 \mu \mathrm{L}$ cDNA template, and 2.4 $\mu \mathrm{L} \mathrm{ddH_{2 }}$ O. The qRT-PCR reactions were performed with CFX96 ${ }^{\text {TM }}$ Real-Time PCR Detection System (Bio-Rad, USA). The gene primers used for qPCR are listed in Table S6. The reactions for each gene were conducted in triplicate with the thermal cycling conditions as follows: $95^{\circ} \mathrm{C}$ for $30 \mathrm{~s}$, followed by 40 cycles of $95^{\circ} \mathrm{C}$ for $5 \mathrm{~s}$ and $57^{\circ} \mathrm{C}$ for $30 \mathrm{~s}$. The primer specificity was confirmed by melting curve analysis. Relative expression levels of the genes were calculated using the $2^{-}$ $\triangle \triangle C T$ method following previous study [56].

\section{Abbreviations}

qRT-PCR: quantitative real-time PCR ABA: abscisic acid JAs: Jasmonates

AdPDC1: Actinidia deliciosa pyruvate decarboxylase $1 \quad$ IAA: Indole acetic acid

GA: Gibberellin PSIl: Photosystem II HR: Hypersensitive responses

DEGs: different expression genes WL: Waterlogging CK: Normal conditions

DMA: Dry matter accumulation CCDs: Carotenoid cleavage dioxygenases

GO: Gene Ontology SCX: Strong cation exchange

LC-MS/MS: Liquid chromatography-tandem mass spectrometry

\section{Declarations}

\section{Ethics approval and consent to participate}

Not applicable.

\section{Consent to publish}

Not applicable.

\section{Availability of data and materials}

The raw data of protein will be deposited into the PRIDE database prior to publication. The proteins identified in this study has been deposited into FigShare with https://doi.org/10.6084/m9.figshare.10026560.v1; the protein expression data has been deposited into FigShare with https://doi.org/10.6084/m9.figshare.10026548.v1.

\section{Competing interests}

The authors declare that they have no competing interests.

\section{Funding}

This work was supported by the National Key Research and Development Program of China (No. 2016YFD0300107, No. 2017YFD0300202-3) and the National Natural Science Foundation of China (No. 31371580, No. 31871578). 


\section{Authors' Contributions}

XW and AS designed the experiments. MW conducted iTRAQ experiments. LL conducted biologic information analysis. RY conducted qRT-PCR. KX conducted waterlogging phenotype collection. MW, LL, and KX wrote the manuscript.

\section{Acknowledgments}

Not applicable.

\section{References}

1. Ahmed F, Rafii M, Ismail M, Juraimi A, Rahim H, Asfaliza R, Latif M. Waterlogging tolerance of crops: breeding, mechanism of tolerance, molecular approaches, and future prospects. Biomed Res Int. 2013; 963525.

2. Herzog M, Striker G, Colmer T, Pedersen O. Mechanisms of waterlogging tolerance in wheat-a review of root and shoot physiology. Plant Cell Environ. 2016; 39(5): 1068-86.

3. Ghobadi ME, Ghobadi M, Zebarjadi A. Effect of waterlogging at different growth stages on some morphological traits of wheat varieties. Int J Biometeorol. 2017; 67.

4. Nguyen T, Tuan P, Mukherjee S, Son S, Ayele B. Hormonal regulation in adventitious roots and during their emergence under waterlogged conditions in wheat. J Exp Bot. 2018; 69(16): 4065-82.

5. Bailey-Serres J, Lee SC, Brinton E. Waterproofing crops: effective flooding survival strategies. Plant Physiol. 2012; 160: 1698-

6. Yamauchi T, Colmer TD, Pedersen O, Nakazono M. Regulation of root traits for internal aeration and tolerance to soil waterlogging-flooding stress. Plant Physiol. 2018; 176(2):1118-30.

7. Nishiuchi S, Yamauchi T, Takahashi H, Kotula L, Nakazono M. Mechanisms for coping with submergence and waterlogging in rice. Rice. 2012; 5: 2.

8. Christianson J, Llewellyn D, Dennis E, Wilson I. Global Gene Expression Responses to Waterlogging in Roots and Leaves of Cotton (Gossypium hirsutum L.). Plant Cell Physiol. 2010; 51(1): 21-37.

9. Zhang Y, Kong X, Dai J, Luo Z.; Li Z, Lu H, Xu S, Tang W, Zhang D, Li W, Xin C, Dong H. Global gene expression in cotton (Gossypium hirsutum L.) leaves to waterlogging stress. Plos One. 2017; 12(9): e0185075.

10. Lee YH, Kim KS, Jang YS, Hwang JH, Lee DH, Choi IH. Global gene expression responses to waterlogging in leaves of rape seedlings. Plant Cell Rep. 2014; 33: 289-99.

11. Du HW, Zhu JX, Su H, Huang M, Wang HW, Ding SC, Zhang BL, Luo A, Wei SD, Tian XH, Xu YB. Bulked segregant RNA-seq reveals differential expression and snps of candidate genes associated with waterlogging tolerance in maize. Front Plant Sci. 2017; 8: 1022.

12. Arora K, Panda KK, Mittal S, Mallikarjuna MG, Rao AR, Dash PK, Thirunavukkarasu N. RNAseq revealed the important gene pathways controlling adaptive mechanisms under waterlogged stress in maize. Scientific Reports. 2017; 7: 10950

13. Qi XH, Xu XW, Lin XJ, Zhang WJ, Chen XH. Identification of differentially expressed genes in cucumber (Cucumis sativus L.) root under waterlogging stress by digital gene expression profi Genomics. 2012; 99: 160-8.

14. Ahsana N, Lee DG, Lee SH, Kang KY, Bahk JD, Choi MS, Lee IJ, Renaut J, Lee BH. A comparative proteomic analysis of tomato leaves in response to waterlogging stress. Physiol Plant. 2007; 131: 555-0.

15. Xu XW, Ji J, Ma XT, Xu Q, Qi XH, Chen XH. Comparative proteomic analysis provides insight into the key proteins involved in cucumber (Cucumis sativus L.) adventitious root emergence under waterlogging stress. Front Plant Sci. 2016; 7: 1515

Page $10 / 20$ 
16. Luan HY, Shen HQ, Pan YH, Guo BJ, Lv C, Xu RG. Elucidating the hypoxic stress response in barley (Hordeum vulgare L.) during waterlogging: A proteomics approach. Scientific Reports. 2018; 8: 9655.

17. Zhang JY, Huang SN, Wang G, Xuan JP, Guo ZR. Overexpression of Actinidia deliciosa pyruvate decarboxylase 1 gene enhances waterlogging stress in transgenic Arabidopsis thaliana. Plant Physiology and Biochemistry. 2016; 106: $244 \mathrm{e} 252$.

18. Li CY, Jiang D, Wollenweber B, Li Y, Dai TB, Cao WX. Waterlogging pretreatment during vegetative growth improves tolerance to waterlogging after anthesis in wheat. Plant Science. 2011; 180: 672-8.

19. Nguyen TN, Son SH, Jordan MC, Levin DB, Ayele BT. Lignin biosynthesis in wheat (Triticum aestivum L.): its response to waterlogging and association with hormonal levels. BMC Plant Biology. 2016; 16: 28.

20. Hamonts K, Clough TJ, Stewart A, Clinton PW, Richardson AE, Wakelin SA, O'Callaghan M, Condron LM. Effect of nitrogen and waterlogging on denitrifier gene abundance, community structure and activity in the rhizosphere of wheat. FEMS Microbiol Ecolo. 2013; 83: 568-84.

21. Pan R, He D, Xu L, Zhou M, Li C, Wu C, et al. Proteomic analysis reveals response of differential wheat (Triticum aestivum L.) genotypes to oxygen deficiency stress. BMC genomics 2019; 20(1), 60.

22. Jiang D, Fan XM, Dai TB, Cao Nitrogen fertiliser rate and post-anthesis waterlogging effects on carbohydrate and nitrogen dynamics in wheat. Plant Soil. 2008; 304: 301-14.

23. Komatsu S, Hiraga S, Yanagawa Y. Proteomics Techniques for the Development of Flood Tolerant Crops. J Proteome Res. 2012; 11: 68-78.

24. Alam I, Lee DG, Kim KH, Park CH, Sharmin SA, Lee H, Oh KW, Yun BW, Lee BH. Proteome analysis of soybean root under waterlogging stress at an early vegitatibe stage. J Biosci. 2010; 35: 49-62.

25. Komatsu S, Kamal AHM, Hossain Wheat proteomics: proteome modulation and abiotic stress acclimation. Front Plant Sci. 2014; 5: 684

26. Romina P, Abeledo LG, Miralles DJ. Identifying the critical period for waterlogging on yield and its components in wheat and barley. Plant Soil. 2014; 378: 265-77.

27. Lina J, Shan X, Jiang C, Hongjian G, Ligan Z. The effect of persistent flooding on the kinetic nutrient absorption and output of wheat. J Chinese Agricultural Science Bulletin. 2012; 28(27): 113-7.

28. Ahsan N, Lee DG, Lee SH, Kang KY, Bahk JD, Choi MS, Lee IJ, Renaut J, Lee BH. A comparative proteomic analysis of tomato leaves in response to waterlogging stress. Physiol Plant. 2007; 131: 555-70.

29. Yan SP, Zhang QY, Tang ZC, Su WA, Sun WN. Comparative proteomic analysis provides new insight into chilling stress response in rice. Mol Cell Proteomics. 2006; 5: 484-96.

30. Mano Y, Omori F. Flooding tolerance in interspecific introgression lines containing chromosome segments from teosinte (Zea nicaraguensis) in maize (Zea mays subsp.mays). Annals of Botany. 2013; 112: 1125-39.

31. Ding JF, Su SN, Liang P. Effect of waterlogging at elongation or after anthesis on grain yield and remobilization of dry matter and nitrogen in wheat. Journal of Triticeae Crops. 2017; 37(11): 1473-79.

32. Araki H, Hamada A, Hossain M, Takahashi T. Waterlogging at jointing and/or after anthesis in wheat induces early leaf cence and impairs grain filling. Field Crops Research. 2012; 137: 27-36.

33. Li CY, Cai J, Jiang D, Dai TB, Cao WX. Effects of hardening by pre-anthesis waterlogging on grain yield and quality of post-anthesis waterlogging wheat (Triticum aestivum L. cv Yangmai 9). Acta Ecologica Sinica. 2011; 31: 1904-10.

34. Zeng N, Yang Z, Zhang Z, Hu L, Chen L. Comparative Transcriptome Combined with Proteome Analyses Revealed Key Factors Involved in Alfalfa (Medicago sativa) Response to Waterlogging Stress. International journal of molecular sciences 2019; 20: 1359.

35. Luan H, Shen H, Pan Y, Guo B, Lv C, Xu R. Elucidating the hypoxic stress response in barley (Hordeum vulgare) during waterlogging: A proteomics approach. Scientific reports 2018; 8: 9655. 
36. Ahsan N, Lee DG, Lee, SH, Kang KY, Bahk JD, Choi MS, Lee IJ, Renaut J, Lee BH. A comparative proteomic analysis of tomato leaves in response to waterlogging stress. Physiologia Plantarum 2007; 131: 555-570.

37. Wang X, Khodadadi E, Fakheri B, Komatsu S. Organ-specific proteomics of soybean seedlings under flooding and drought stresses. Journal of proteomics 2017; 162: 62-72.

38. Wang X, Huang M, Zhou Q, Cai J, Dai T, Cao W, Jiang D. Physiological and proteomic mechanisms of waterlogging priming improves tolerance to waterlogging stress in wheat (Triticum aestivum). Environmental and Experimental Botany, 2016; 132, 175-182.

39. Kong FJ, Oyanagi A, Komatsu S. Cell wall proteome of wheat roots under flooding stress using gel-based and LC MS/MS-based proteomics approaches. Biochimica et Biophysica Acta (BBA)-Proteins and Proteomics2010; 1804 : 124-136.

40. Beinert H, Holm RH, Münck E. Iron-sulfur clusters: nature's modular, multipurpose structures. Science. 1997; 277: 6539.

41. Lill R. Function and biogenesis of iron-sulphur proteins. Nature. 2009; 460: 831-8.

42. Sasaki S, Minamisawa K, Mitsui H. A Sinorhizobium meliloti RpoH-Regulated Gene Is Involved in Iron-Sulfur Protein Metabolism and Effective Plant Symbiosis under Intrinsic Iron Limitation. J Bacteriol. 2016; 198: 2297-306.

43. Khan MS, Khraiwesh B, Pugalenthi G, Gupta RS, Singh J, Duttamajumder SK, Kapur R. Subtractive hybridizationmediated analysis of genes and in silico prediction of associated microRNAs under waterlogged conditions in sugarcane (Saccharum spp.). FEBS Open Bio. 2014; 4: 533-41.

44. Zeng Y, Chung K, Li B, Lai C, Lam S, Wang X, Cui Y, Gao C, Luo M, Wong K, Schekman R, Jiang L. Unique COPII component AtSar1a/AtSec23a pair is required for the distinct function of protein ER export in Arabidopsis thaliana. Proc Natl Acad Sci USA. 2015; 112(46): 14360-5.

45. Singh AK, Kumar R, Pareek A, Sopory SK, Singla-Pareek SL. Overexpression of rice CBS domain containing protein improves salinity, oxidative, and heavy metal tolerance in transgenic tobacco. Mol Biotechnol. 2012; 52: 205-16.

46. Hao Q, Shang W, Zhang C, Chen H, Chen L, Yuan S, Chen S, Zhang X, Zhou Identification and Comparative Analysis of CBS Domain-Containing Proteins in Soybean (Glycine max) and the Primary Function of GmCBS21 in Enhanced Tolerance to Low Nitrogen Stress. Int J Mol Sci. 2016; 17(5): 620.

47. Dennis ES, Dolferus $R$, Ellis $M$, Rahman M, Wu Y, Hoeren FU, et al. Molecular strategies for improving waterlogging tolerance in plants. Journal of experimental botany 2000; 51(342), 89-97.

48. Kumutha D, Sairam RK, Ezhilmathi K, Chinnusamy V, Meena RC. Effect of waterlogging on carbohydrate metabolism in pigeon pea (Cajanus cajan L.): upregulation of sucrose synthase and alcohol dehydrogenase. Plant Science, 2008; 175(5), 706-716.

49. Vinyard DJ, Ananyev GM, Dismukes GM. Photosystem II: The Reaction Center of Oxygenic Photosynthesis. Annu Rev Biochem. 2013; 82: 577-606

50. Pan XY, Zheng HY, Zhao JY, Xu YJ, Li X ZmCCD7/ZpCCD7 encodes a carotenoid cleavage dioxygenase mediating shoot branching. Planta. 2016; 243(6): 1407-18.

51. Rubio-Moraga A, Rambla JL, Fernández-de-Carmen A, Trapero-Mozos A, Ahrazem O, Orzáez D, Granell A, GómezGómez L. New target carotenoids for CCD4 enzymes are revealed with the characterization of a novel stress-induced carotenoid cleavage dioxygenase gene from Crocus sativus. Plant Mol Biol. 2014; 86(4-5): 555-69.

52. Baek K, Seo PJ, Park CM. Activation of a Mitochondrial ATPase Gene Induces abnormal Seed Development in Arabidopsis. Mol Cells. 2011; 31(4): 361-9.

53. Ksas B, Becuwe N, Chevalier A, Havaux M. Plant tolerance to excess light energy and photooxidative damage relies on plastoquinone biosynthesis. Sci Rep. 2015; 5: 10919.

Page $12 / 20$ 
54. Gong W, Xu F, Sun J, Peng Z, He S, Pan Z, Du X. iTRAQ-Based Comparative Proteomic Analysis of Seedling Leaves of Two Upland Cotton Genotypes Differing in Salt Tolerance. Front Plant Sci. 2017; 8: 2113.

55. Xie C, Mao X, Huang J, Ding Y, Wu J, Dong S, Kong L, Gao G, Li C, Wei L. KOBAS 2.0: a web server for annotation and identification of enriched pathways and diseases. Nucleic acids research 2011; 39: W316-W322.

56. Kim J, Nguyen N, Nguyen N, Hong S, Lee H. Loss of all three calreticulins, CRT1, CRT2 and CRT3, causes enhanced sensitivity to water stress in Arabidopsis. Plant Cell Rep. 2013; 32: 1843-3.

\section{Tables}

Table 1 Effect of waterlogging on yield and yield components of wheat

\begin{tabular}{|c|c|c|c|c|c|c|c|}
\hline Cultivar & Treatment & $\begin{array}{l}\text { DMA1 } \\
\text { (g stem- } \\
\text { 1) }\end{array}$ & $\begin{array}{l}\text { DMA2 } \\
\text { (g stem- } \\
\text { 1) }\end{array}$ & $\begin{array}{l}\text { Kernel per } \\
\text { spike }\end{array}$ & $\begin{array}{l}\text { 1000-kernel weight } \\
\text { (g) }\end{array}$ & $\begin{array}{l}\text { Grain yield } \\
\text { weight } \\
\text { (g stem-1) }\end{array}$ & $\begin{array}{l}\text { Harvest } \\
\text { index }\end{array}$ \\
\hline \multirow[t]{3}{*}{ XM55 } & CK & $2.07 a$ & $3.21 b$ & 43.31b & $29.33 b$ & $1.41 b$ & $0.44 b$ \\
\hline & WL & $2.1 \mathrm{a}$ & $2.81 \mathrm{c}$ & 41.87b & $24.02 c$ & $1.04 \mathrm{c}$ & $0.37 c$ \\
\hline & $\begin{array}{l}(\mathrm{WL}- \\
\mathrm{CK}) / \mathrm{CK}\end{array}$ & / & 0.125 & 0.033 & 0.181 & 0.262 & 0.159 \\
\hline \multirow[t]{3}{*}{ YM158 } & CK & $2.19 a$ & $3.57 a$ & $45.47 a$ & $34.47 a$ & $1.63 a$ & $0.46 a$ \\
\hline & WL & $2.17 a$ & $2.84 \mathrm{c}$ & $41.56 b$ & $25.31 \mathrm{c}$ & $1.03 c$ & $0.36 c$ \\
\hline & $\begin{array}{l}(\mathrm{WL}- \\
\mathrm{CK}) / \mathrm{CK}\end{array}$ & / & 0.205 & 0.108 & 0.362 & 0.368 & 0.218 \\
\hline
\end{tabular}

The lowercase letters indicate significant differences at $\mathrm{P}<0.05$ among treatments as determined by Duncan's Multiple Range Test. DMA1, aboveground dry matter accumulation at anthesis before waterlogging $\triangle \mathrm{DMA} 2$, aboveground dry matter accumulation at maturity; CK, Control; WL, Waterlogging

Table 2. Differentially expressed proteins between XM55 and YM158 under WL 
Gene ID

$\log 2$ FC

Protein Description

Functional

(XM55/YM158)

Category

UP-regulated

TRIAE_CS42_2BL_TGACV1_130584_AA0414140.1 1.63

TRIAE_CS42_2BL_TGACv1_131439_AA0427700.2 1.50

Ubiquinol oxidase $4 \quad$ redox

Superoxide dismutase redox

TRIAE_CS42_4BL_TGACv1_321826_AA1065960.1 1.18

[Mn],

TRIAE_CS42_3AL_TGACv1_195570_AA0651350.1 0.33

heat shock protein $101 \quad$ stress response

TRIAE_CS42_3AL_TGACv1_195570_AA0651350.2 0.33

Fe-S cluster assembly

factor HCF101

chloroplast

Fe-S cluster assembly chloroplast

TRIAE_CS42_3AL_TGACv1_195570_AA0651350.3 0.33 factor HCF101

Fe-S cluster assembly chloroplast factor HCF101

TRIAE_CS42_3DL_TGACv1_250912_AA0874940.1 0.33

Fe-S cluster assembly chloroplast factor HCF101

TRIAE_CS42_3DL_TGACv1_250912_AA0874940.2 0.33

Fe-S cluster assembly chloroplast factor HCF101

TRIAE_CS42_2BL_TGACv1_131039_AA0421600.2 0.32

heat shock cognate $70 \quad$ transcription kDa protein 2-like

TRIAE_CS42_6DL_TGACv1_526647_AA1688990.1 0.32

heat shock cognate $70 \quad$ transcription kDa protein 2-like

TRIAE_CS42_3AS_TGACv1_211332_AA0688720.1 0.31

GTP-binding protein SAR1A-like

GTP binding

GTP-binding protein

SAR1A-like

GTP binding

TRIAE_CS42_3DS_TGACv1_272355_AA0919480.1 0.31

CBS domain-containing

TRIAE_CS42_6DL_TGACv1_526455_AA1684150.2 0.29

protein

TRIAE_CS42_6DL_TGACv1_526455_AA1684150.3 0.29

CBS domain-containing

protein

Down-regulated

$\begin{array}{ll}\text { AIG90456 } & -0.26\end{array}$

photosystem II reaction plastid center protein $\mathrm{H}$

TRIAE_CS42_5DS_TGACv1_456540_AA1473460.1 - 0.36 carotenoid 9,10(9',10') cleavage dioxygenaselike

TRIAE_CS42_4BS_TGACv1_330468_AA1107820.2 -0.67 psbP-like protein 1, chloroplastic

TRIAE_CS42_4BS_TGACv1_329474_AA1101780.1 -0.87 mitochondrial ATPase inhibitor

TRIAE_CS42_4BS_TGACv1_329474_AA1101780.3 -0.87

TRIAE_CS42_2BL_TGACv1_132610_AA0438610.1 -1.29 mitochondrial ATPase photorespiration inhibitor stress response Aminomethyltransferase redox

TRIAE_CS42_6BL_TGACv1_503168_AA1627380.1 -0.78 Uncharacterized protein 


\section{Additional File Legends}

Additonal file 1: Table S1. Differentially expressed proteins between XM55 and YM158 under control Additonal file 2: Table S2. Differentially expressed proteins in XM55 between WL and CK Additonal file 3: Table S3. Differentially expressed proteins in YM158 between WL and CK Additonal file 4: Table S4. GO annotation of differntial expressed proteins Additonal file 5: Table S5. KO annotation of differntial expressed proteins Additonal file 6: Table S6. qRT-PCR primers for analysis of mRNA expression Additional file 7: Table S7. The full list of proteins identified in this study. Additional file 8: Figure S1. GO annotation of differentially expressed proteins between WL and CK in XM55 Additonal file 9: Figure S2. GO annotation of differentially expressed proteins between WL and CK in YM158 Additonal file 10: Figure S3. The expression levels of three candidate genes detected using RT-qPCR method Additonal file 11: Figure S4. SDS-PAGE analysis for the samples.

\section{Figures}



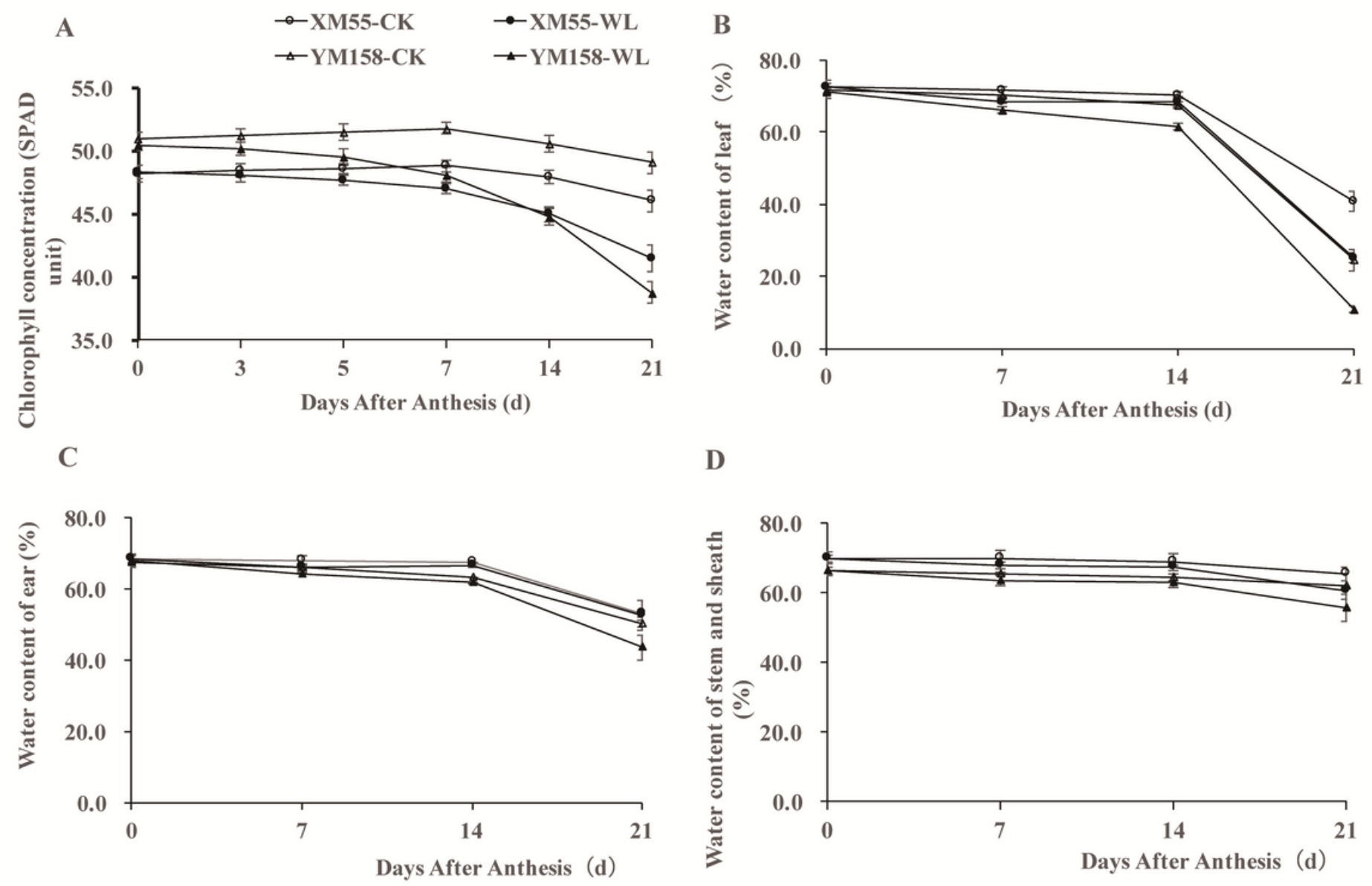

D

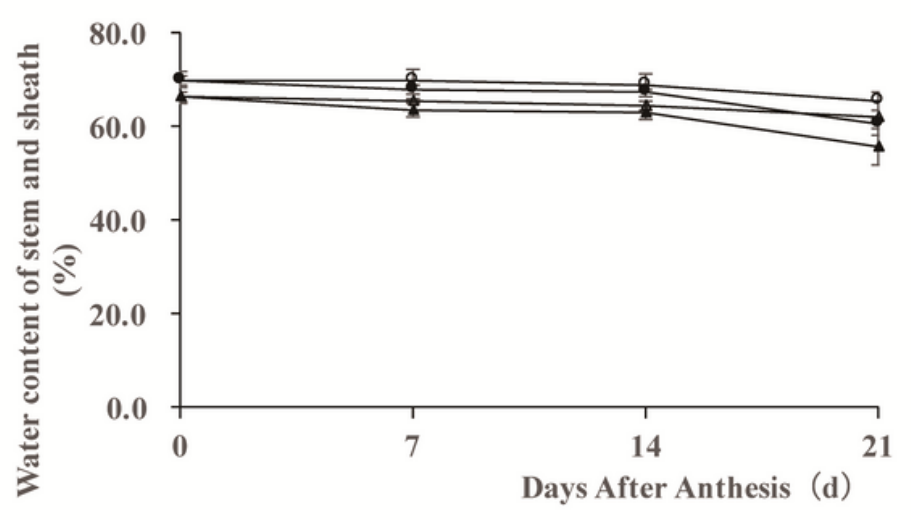

\section{Figure 1}

Phenotypes of XM55 and YM158 under waterlogging and control conditions. (A) dynamic changes of chlorophyll concentration (SPAD unit) of the last expanded leaf after 7 days waterlogging at anthesis; (B) dynamic changes of water content of leaf after waterlogging at anthesis between different varieties; (C) dynamic changes of ear water content after waterlogging at anthesis between different varieties; (D) dynamic changes of water content of stem and sheath after waterlogging at anthesis between different varieties. 
A

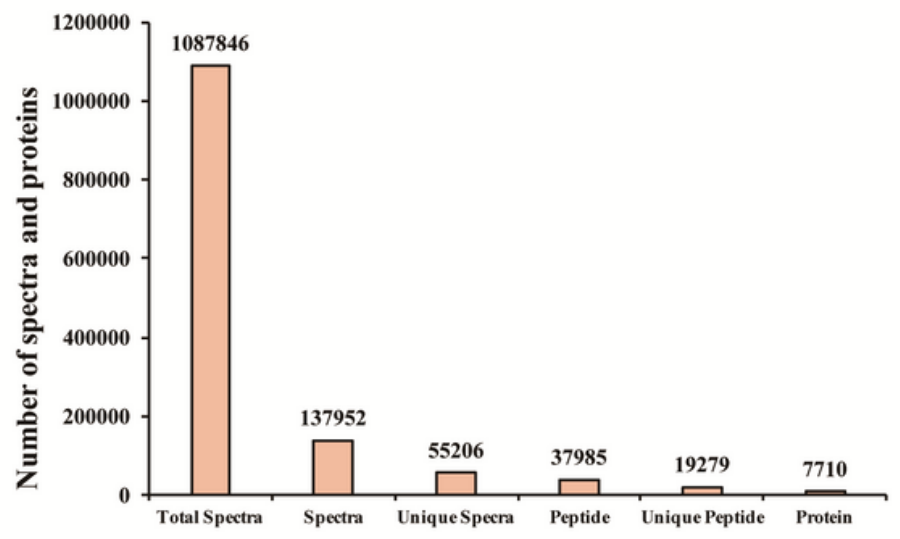

B

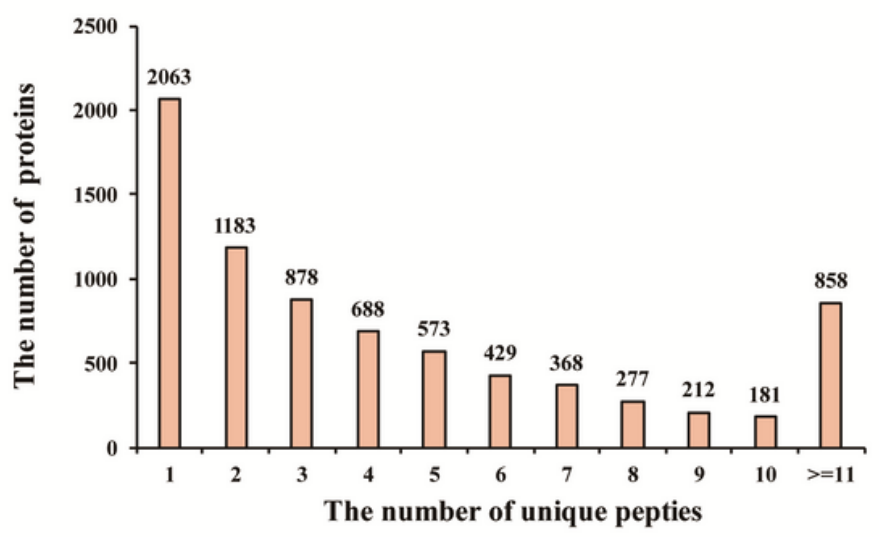

Figure 2

Mass spectrometry analysis and protein identification. A, the number of spectra and proteins. B, the number of proteins with different unique peptides.

A

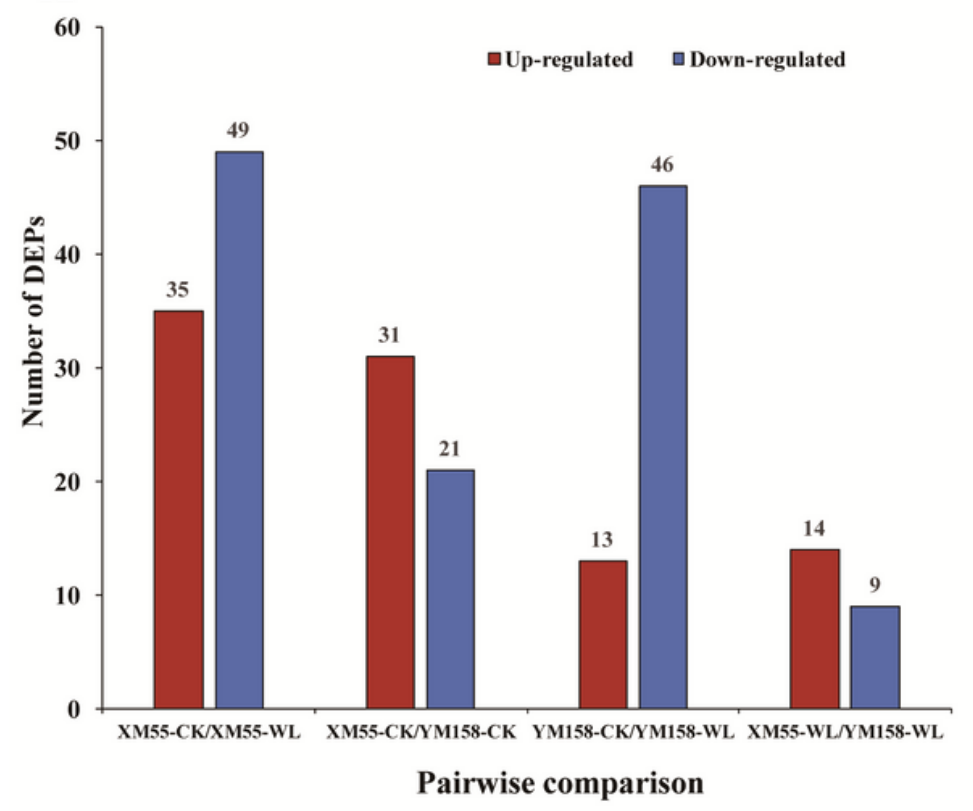

B

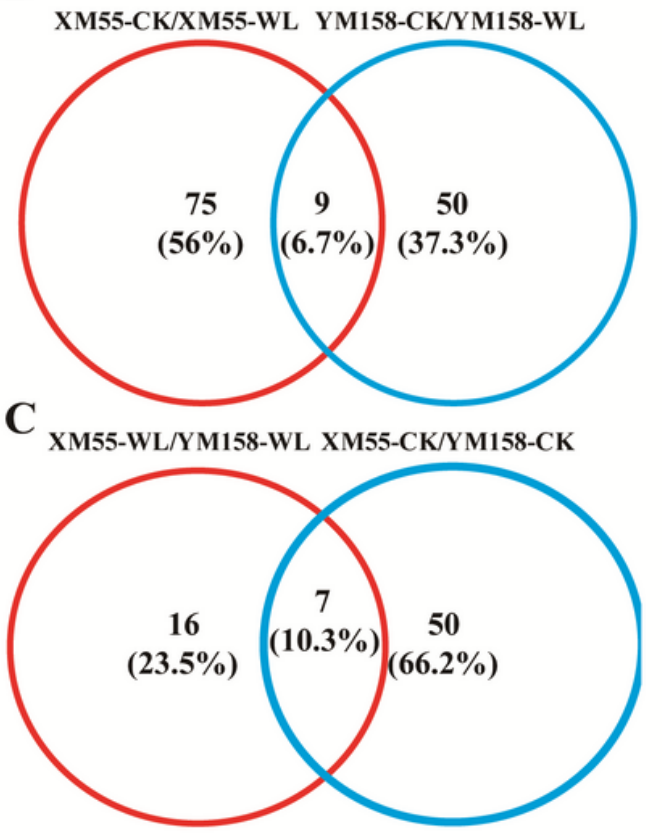

Figure 3

Quantitative and Venn analysis of the proteome of two wheat cultivars under different treatments. (A) Quantitative analysis of the proteome between the waterlogging treated and control samples; (B) Venn analysis of two wheat cultivars under different treatments; (C) Venn analysis of different treatments in different wheat cultivars; XM55 and YM158 are the two cultivars; CK, control; WL, waterlogging 


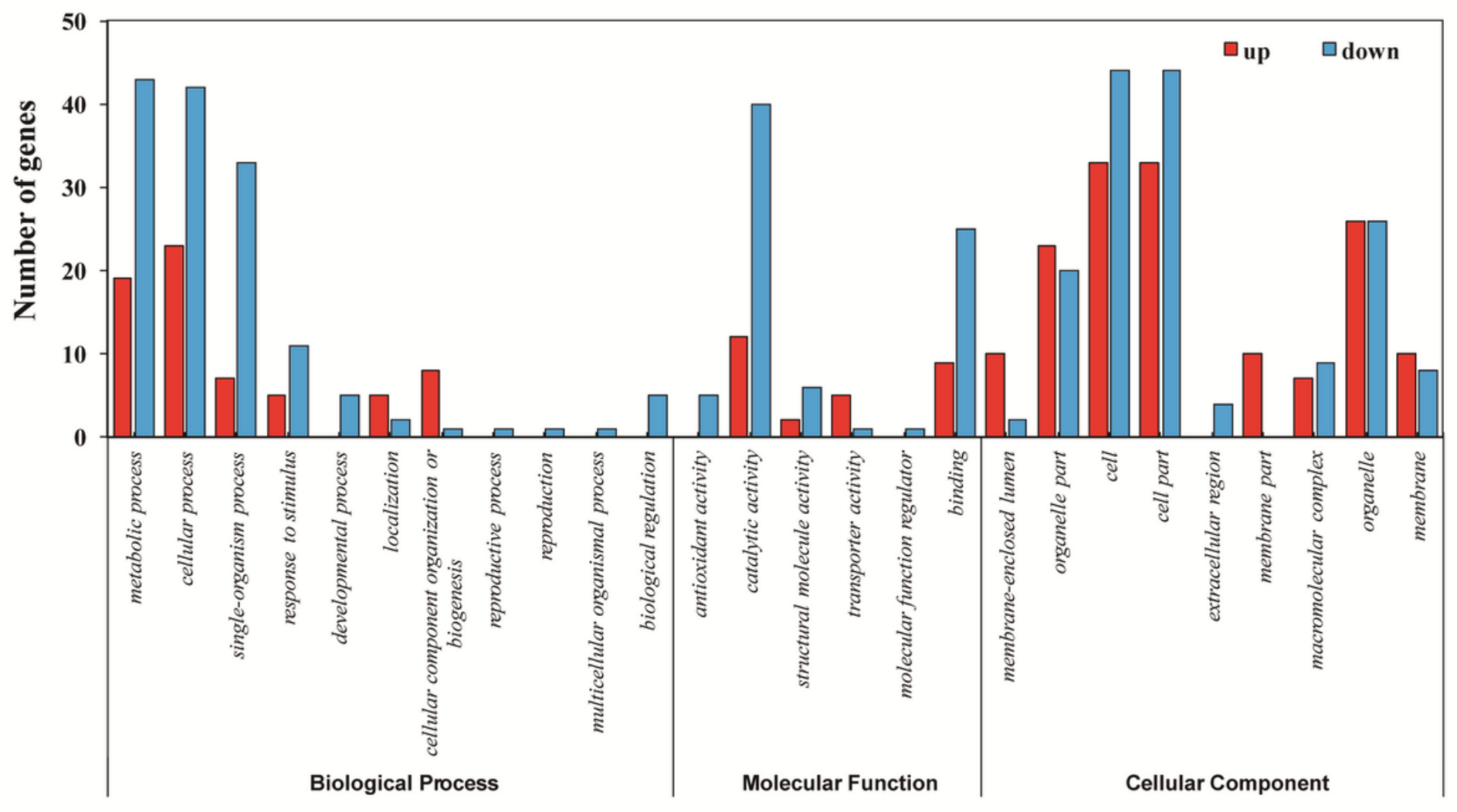

Figure 4

G0 annotation of differentially expressed proteins between XM55 and YM158 under WL 

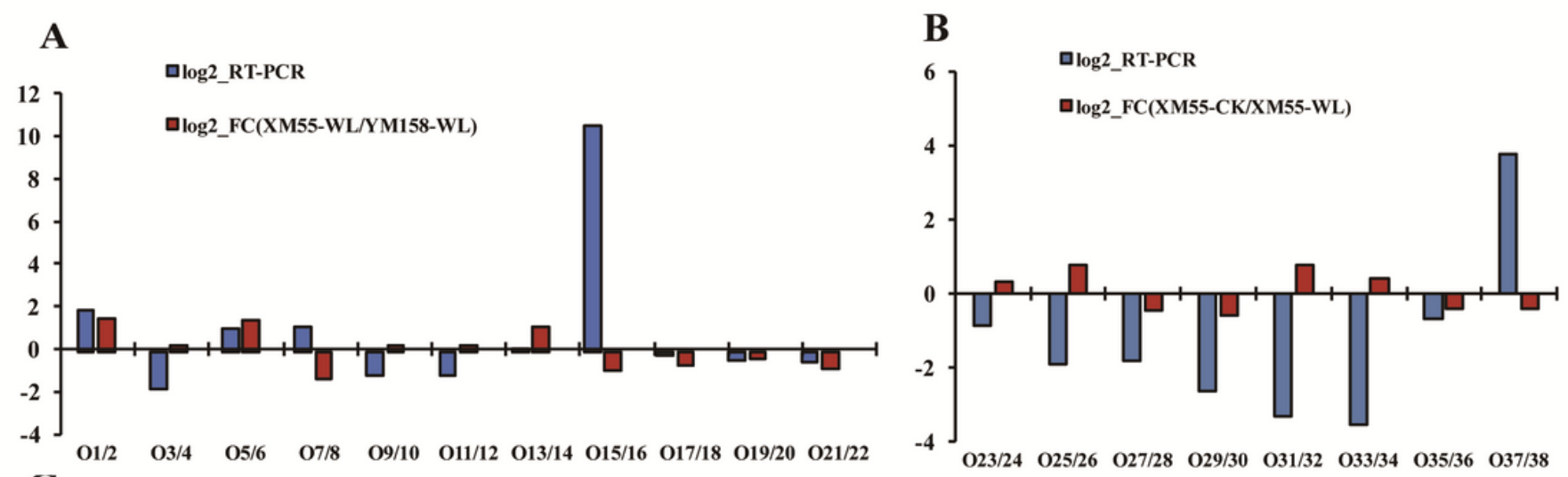
C

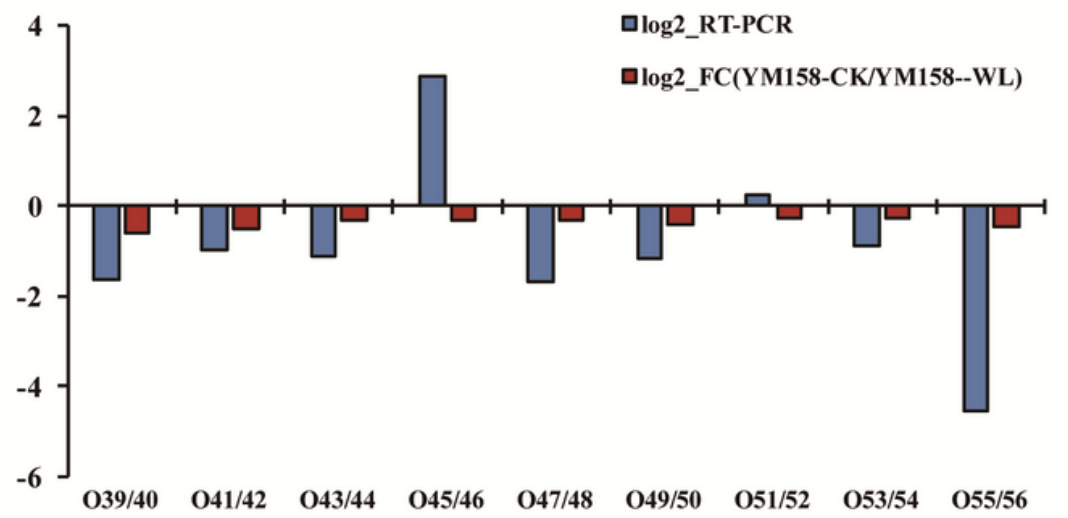

Figure 5

Correlation of differentially expressed protein at transcript and translation level. Differences in protein expression and qRT-PCR between XM55 and YM158 under waterlogging stress (A) Differences in protein expression measured by iTRAQ and quantitative real-time reverse transcription-PCR (qRT-PCR) in XM55 (B) and YM158 (C) under WL and CK. Log2-RTPCR represents RNA expression level; Log2-FC represents the differences in protein expression level.

\section{Supplementary Files}

This is a list of supplementary files associated with this preprint. Click to download.

- SupplementaryFigureS1.jpg

- SupplementaryFigureS3.jpg

- SupplementaryTableS7.xlsx

- SupplementaryTableS1.docx

- SupplementaryTableS3.docx

- SupplementaryTableS5.xlsx

- SupplementaryTableS6.docx

- SupplementaryFigureS2.jpg

- SupplementaryFigureS4.jpg

- SupplementaryTables4.xlsx 
- SupplementaryTableS2.docx

Page 20/20 